\title{
The Effect of Different Writing Tasks on Linguistic Style: A Case Study of the ROC Story Cloze Task
}

\author{
Roy Schwartz ${ }^{1,2}$, Maarten Sap ${ }^{1}$, Ioannis Konstas ${ }^{1}$, \\ Li Zilles ${ }^{1}$, Yejin Choi ${ }^{1}$ and Noah A. Smith ${ }^{1}$ \\ ${ }^{1}$ Paul G. Allen School of Computer Science \& Engineering, \\ University of Washington, Seattle, WA, USA \\ ${ }^{2}$ Allen Institute for Artificial Intelligence, Seattle, WA, USA \\ \{roysch, msap, ikonstas, lzilles, yejin, nasmith\}@cs.washington.edu
}

\begin{abstract}
A writer's style depends not just on personal traits but also on her intent and mental state. In this paper, we show how variants of the same writing task can lead to measurable differences in writing style. We present a case study based on the story cloze task (Mostafazadeh et al., 2016a), where annotators were assigned similar writing tasks with different constraints: (1) writing an entire story, (2) adding a story ending for a given story context, and (3) adding an incoherent ending to a story. We show that a simple linear classifier informed by stylistic features is able to successfully distinguish among the three cases, without even looking at the story context. In addition, combining our stylistic features with language model predictions reaches state of the art performance on the story cloze challenge. Our results demonstrate that different task framings can dramatically affect the way people write. ${ }^{1}$
\end{abstract}

\section{Introduction}

Writing style is expressed through a range of linguistic elements such as words, sentence structure, and rhetorical devices. It is influenced by personal factors such as age and gender (Schler et al., 2006), by personality traits such as agreeableness and openness (Ireland and Mehl, 2014), as well as by mental states such as sentiment (Davidov et al., 2010), sarcasm (Tsur et al., 2010), and deception (Feng et al., 2012). In this paper, we study the extent to which writing style is affected by the nature of the writing task the writer was asked to perform,

\footnotetext{
${ }^{1}$ This paper extends our LSDSem 2017 shared task submission (Schwartz et al., 2017).
}

\begin{tabular}{|c|c|}
\hline Story Prefix & Ending \\
\hline \multirow{3}{*}{$\begin{array}{l}\text { John liked a girl at his } \\
\text { work. He tried to get } \\
\text { her attention by acting } \\
\text { silly. She told him to } \\
\text { grow up. John con- } \\
\text { fesses he was trying } \\
\text { to make her like him } \\
\text { more. }\end{array}$} & $\begin{array}{l}\text { She feels flattered } \\
\text { and asks John on a } \\
\text { date. }\end{array}$ \\
\hline & $\begin{array}{l}\text { The girl found this } \\
\text { charming, and gave } \\
\text { him a second chance. }\end{array}$ \\
\hline & $\frac{\text { John was happy about }}{\text { being rejected. }}$ \\
\hline
\end{tabular}

Table 1: Examples of stories from the story cloze task. The table shows a story prefix with three contrastive endings: The original ending, a coherent ending and a incoherent one.

since different tasks likely engage different cognitive processes (Campbell and Pennebaker, 2003; Banerjee et al., 2014). ${ }^{2}$

We show that similar writing tasks with different constraints on the author can lead to measurable differences in her writing style. As a case study, we present experiments based on the recently introduced ROC story cloze task (Mostafazadeh et al., 2016a). In this task, authors were asked to write five-sentence self-contained stories, henceforth original stories. Then, each original story was given to a different author, who was shown only the first four sentences as a story context, and asked to write two contrasting story endings: a right (coherent) ending, and a wrong (incoherent) ending. Framed as a story cloze task, the goal of this dataset is to serve as a commonsense challenge for NLP and AI research. Table 1 shows an example of an original story, a coherent story, and an incoherent story.

While the story cloze task was originally de-

\footnotetext{
${ }^{2}$ For the purposes of this paper, style is defined as contentagnostic writing characteristics, such as the number of words in a sentence.
} 
signed to be a story understanding challenge, its annotation process introduced three variants of the same writing task: writing an original, right, or wrong ending to a short story. In this paper, we show that a linear classifier informed by stylistic features can distinguish among the different endings to a large degree, even without looking at the story context (64.5-75.6\% binary classification results).

Our results allow us to make a few key observations. First, people adopt a different writing style when asked to write coherent vs. incoherent story endings. Second, people change their writing style when writing the entire story on their own compared to writing only the final sentence for a given story context written by someone else.

In order to further validate our method, we also directly tackle the story cloze task. Adapting our classifier to the task, we obtain $72.4 \%$ accuracy, only $2.3 \%$ below state of the art results. We also show that the style differences captured by our model can be combined with neural language models to make a better use of the story context. Our final model that combines context with stylistic features achieves a new state of the art-75.2\% - an additional $2.8 \%$ gain.

The contributions of our study are threefold. First, findings from our study can potentially shed light on how different kinds of cognitive load influence the style of written language. Second, combined with recent similar findings of Cai et al. (2017), our results indicate that when designing new NLP tasks, special attention needs to be paid to the instructions given to authors. Third, we establish a new state of the art result on the commonsense story cloze challenge. Our code is available at https://github.com/ roys174/writing_style.

\section{Background: The Story Cloze Task}

To understand how different writing tasks affect writing style, we focus on the story cloze task (Mostafazadeh et al., 2016a). While this task was developed to facilitate representation and learning of commonsense story understanding, its design included a few key choices which make it ideal for our study. We describe the task below.

ROC stories. The ROC story corpus consists of 49,255 five-sentence stories, collected on Ama- zon Mechanical Turk (AMT). ${ }^{3}$ Workers were instructed to write a coherent self-contained story, which has a clear beginning and end. To collect a broad spectrum of commonsense knowledge, there was no imposed subject for the stories, which resulted in a wide range of different topics.

Story cloze task. After compiling the story corpus, the story cloze task-a task based on the corpus-was introduced. A subset of the stories was selected, and only the first four sentences of each story were presented to AMT workers. Workers were asked to write a pair of new story endings for each story context: one right and one wrong. Both endings were required to complete the story using one of the characters in the story context. Additionally, the endings were required to be "realistic and sensible" (Mostafazadeh et al., 2016a) when read out of context.

The resulting stories, both right and wrong, were then individually rated for coherence and meaningfulness by additional AMT workers. Only stories rated as simultaneously coherent with a right ending and neutral with a wrong ending were selected for the task. It is worth noting that workers rated the stories as a whole, not only the endings.

Based on the new stories, Mostafazadeh et al. (2016a) proposed the story cloze task. The task is simple: given a pair of stories that differ only in their endings, the system decides which ending is right and which is wrong. The official training data contains only the original stories (without alternative endings), while development and test data consist of the revised stories with alternative endings (for a different set of original stories that are not included in the training set). The task was suggested as an extensive evaluation framework: as a commonsense story understanding task, as the shared task for the Linking Models of Lexical, Sentential and Discourse-level Semantics workshop (LSDSem 2017, Roth et al., 2017), and as a testbed for vector-space evaluation (Mostafazadeh et al., 2016b).

Interestingly, only very recently, one year after the task was first introduced, the published benchmark on this task surpassed $60 \%$. This comes in contrast to other recent similar machine reading tasks such as CNN/DailyMail (Hermann et al., 2015), SNLI (Bowman et al., 2015), LAMBADA

\footnotetext{
${ }^{3}$ Recently, additional 53K stories were released, which results in roughly $100 \mathrm{~K}$ stories.
} 
(Paperno et al., 2016) and SQuAD (Rajpurkar et al., 2016), for which results improved dramatically over similar or much shorter periods of time. This suggests that this task is challenging and that high performance is hard to achieve.

In addition, Mostafazadeh et al. (2016a) made substantial efforts to ensure the quality of this dataset. First, each pair of endings was written by the same author, which ensured that style differences between authors could not be used to solve the task. Furthermore, Mostafazadeh et al. implemented nine baselines for the task, using surface level features as well as narrative-informed ones, and showed that each of them reached roughly chance-level. These results suggest that real understanding of text is required in order to solve the task. In this paper, we show that this is not necessarily the case, by demonstrating that a simple linear classifier informed with style features reaches near state of the art results on the task- $72.4 \%$.

Different writing tasks in the story cloze task. Several key design decisions make the task an interesting testbed for the purpose of this study. First, the training set for the task (ROC Stories corpus) is not a training set in the usual sense, ${ }^{4}$ as it contains only positive (right) examples, and not negative (wrong) ones.

On top of that, the original endings, which serve as positive training examples, were generated differently from the right endings, which serve as the positive examples in the development and test sets. While the former are part of a single coherent story written by the same author, the latter were generated by letting an author read four sentences, and then asking her to generate a fifth right ending.

Finally, although the right and wrong sentences were generated by the same author, the tasks for generating them were quite different: in one case, the author was asked to write a right ending, which would create a coherent five-sentence story along with the other four sentences. In the other case, the author was asked to write a wrong ending, which would result in an incoherent five-sentence story.

\section{Surface Analysis of the Story Cloze Task}

We begin by computing several characteristics of the three types of endings: original endings (from

\footnotetext{
${ }^{4}$ I.e., the training instances are not drawn from a population similar to the one that future testing instances will be drawn from.
}

the ROC story corpus training set), right endings and wrong endings (both from the story cloze task development set). Our analysis reveals several style differences between different groups. First, original endings are on average longer (11 words per sentence) than right endings (8.75 words), which are in turn slightly longer than wrong ones (8.47 words). The latter finding is consistent with previous work, which has shown that sentence length is also indicative of whether a text was deceptive (Qin et al., 2004; Yancheva and Rudzicz, 2013). Although writing wrong sentences is not the same as deceiving, it is not entirely surprising to observe similar trends in both tasks.

Second, Figure 1a shows the distribution of five frequent POS tags in all three groups. The figure shows that both original and right endings use pronouns more frequently than wrong endings. Once again, deceptive text is also characterized by fewer pronouns compared to truthful text (Newman et al., 2003).

Finally, Figure $1 b$ presents the distribution of five frequent words across the different groups. The figure shows that original endings use coordinations ("and") more than right endings, and substantially more than wrong ones. Furthermore, original and right endings seem to prefer enthusiastic language (e.g., "!"), while wrong endings tend to use more negative language ("hates"), similar to deceptive text (Newman et al., 2003). Next we show that these style differences are not anecdotal, but can be used to distinguish among the different types of story endings.

\section{Model}

To what extent do different writing constraints lead authors to adopt different writing styles? In order to answer this question, we first use simple methods that have been shown to be very effective for recognizing style (see Section 8). We describe our model below.

We train a logistic regression classifier to categorize an ending, either as right vs. wrong or as original vs. new (right). Each feature vector is computed using the words in one ending, without considering earlier parts of the story. We use the following style features.

- Length: the number of words in the sentence.

- Word n-grams: we use sequences of 1-5 words. Following Tsur et al. (2010) and 


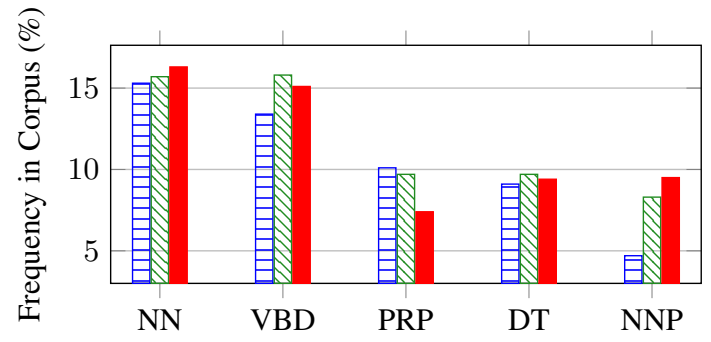

(a) POS tags

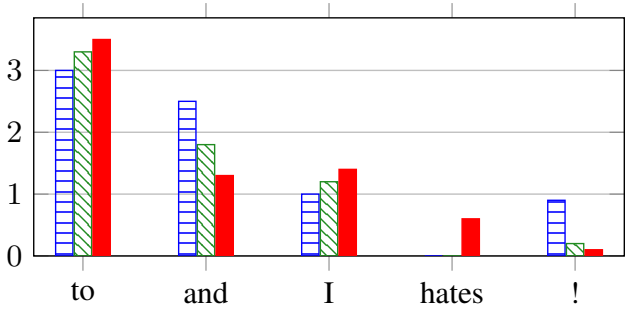

(b) Words

Figure 1: The distribution of five frequent POS tags (1a) and words (1b) across original endings (horizontal lines) from the story cloze training set, and right (diagonal lines) and wrong (solid lines) endings, both from the story cloze task development set.

Schwartz et al. (2013b), we distinguish between high frequency and low frequency words. Specifically, we replace content words (nouns, verbs, adjectives, and adverbs), which are often low frequency, with their part-of-speech tags.

- Character n-grams: character $n$-grams are one of the most useful features in identifying author style (Stamatatos, 2009). We use character 4 -grams. ${ }^{5}$

\section{Experiments}

We design two experiments to answer our research questions. The first is an attempt to distinguish between right and wrong endings, the second between original endings and new (right) endings. For completeness, we also run a third experiment, which compares between original and wrong endings.

Experiment 1: right/wrong endings. The goal of this experiment is to measure the extent to which style features capture differences between the right and wrong endings. As the story cloze task doesn't have a training corpus for the right and wrong endings (see Section 2), we use the development set as our training set, holding out $10 \%$ for development (3,366 training endings, 374 for development). We keep the story cloze test set as is (3,742 endings).

It is worth noting that our classification task is slightly different from the story cloze task. Instead of classifying pairs of endings, one which is right and another which is wrong, our classifier decides about each ending individually, whether it

\footnotetext{
${ }^{5}$ Experiments with 5-grams on our development set reached similar performance.
}

is right (positive instance) or wrong (negative instance). By ignoring the coupling between right and wrong pairs, we are able to decrease the impact of author-specific style differences, and focus on the difference between the styles accompanied with right and wrong writings.

Experiment 2: original/new endings. Here the goal is to measure whether writing the ending as part of a story imposes different style compared to writing a new (right) ending to an existing story. We use the endings of the ROC stories as our original examples and right endings from the story cloze task as new examples. As there are far more original instances than new instances, we randomly select five original sets, each with the same number of instances as we have new instances (3,366 training endings, 374 development endings, and 3,742 test endings). We train five classifiers, one with each of the original training sets, and report the average classification result.

Experiment 3: original/wrong endings. For completeness, we measure the extent to which our classifier can discriminate between original and wrong endings. We replicate Experiment 2, this time replacing right endings with wrong ones.

Experimental setup. In all experiments, we add a START symbol at the beginning of each sentence. ${ }^{6}$ For computing our features, we keep $n$ gram (character or word) features that occur at least five times in the training set. All feature values are normalized to $[0,1]$. For the POS features, we tag all endings with the Spacy POS tagger. ${ }^{7}$ We use Python's sklearn logistic regression imple-

\footnotetext{
${ }^{6} 99 \%$ of all sentences end with a period or an exclamation mark, so we do not add a STOP symbol.

${ }^{7}$ http: //spacy.io/
} 


\begin{tabular}{|l|c|}
\hline Experiment & Accuracy \\
\hline right vs. wrong & 0.645 \\
\hline original vs. right & 0.685 \\
\hline original vs. wrong & 0.756 \\
\hline
\end{tabular}

Table 2: Results of experiments 1 (right vs. wrong), 2 (original vs. right (new)) and 3 (original vs. wrong (new) endings). In all cases, our setup implies a $50 \%$ random baseline.

mentation (Pedregosa et al., 2011) with $L_{2}$ regularization, performing grid search on the development set to tune a single hyperparameter- the regularization parameter.

\subsection{Results}

Table 2 shows our results. In all experiments, our model achieves performance well above what would be expected under chance ( $50 \%$ by design). Noting again that our model ignores the story context (the preceding four sentences), our model is unable to capture any notion of coherence. This finding provides strong evidence that the authors' style was affected by the writing task they were given to perform.

\subsection{Story Cloze Task}

The results of Experiment 1 indicate that right and wrong endings are characterized by different styles. In order to further estimate the quality of our classification results, we tackle the story cloze task using our classifier. This classification task is more constrained than Experiment 1, as two endings are given and the question is which is right and which is wrong. We apply the classifier from Experiment 1 as follows: if it assigns different labels to the two given endings, we keep them. Otherwise, the label whose posterior probability is lower is reversed.

Table 3 shows our results on the story cloze test set. Our classifier obtains $72.4 \%$ accuracy, only $2.3 \%$ lower than state of the art results. Importantly, unlike previous approaches, ${ }^{8}$ our classifier does not require the story corpus training data, and in fact doesn't even consider the first four sentences of the story in question. These numbers further support the claim that the styles of right and wrong endings are indeed very different.

\footnotetext{
${ }^{8}$ One exception is the EndingsOnly system (Cai et al., 2017), which was published in concurrence with this work, and obtains roughly the same results.
}

\begin{tabular}{|l|r|}
\hline Model & Acc. \\
\hline DSSM (Mostafazadeh et al., 2016a) & 0.585 \\
ukp (Mihaylov and Frank, 2017) & 0.711 \\
tbmihaylov (Mihaylov and Frank, 2017) & 0.724 \\
$\dagger \dagger$ EndingsOnly (Cai et al., 2017) & 0.725 \\
cogcomp & 0.744 \\
HIER,ENCPLOTEND,ATT (Cai et al., 2017) & 0.747 \\
\hline \hline RNN & 0.677 \\
$\dagger$ Ours & 0.724 \\
Combined (ours + RNN) & $\mathbf{0 . 7 5 2}$ \\
\hline \hline Human judgment & 1.000 \\
\hline
\end{tabular}

Table 3: Results on the test set of the story cloze task. The middle block are our results. cogcomp results and human judgement scores are taken from Mostafazadeh et al. (2017). Methods marked with $(\dagger)$ do not use the story context in order to make a prediction.

\section{Combination with a neural language model.} We investigate whether our model can benefit from state of the art text comprehension models, for which this task was designed. Specifically, we experiment with an LSTM-based (Hochreiter and Schmidhuber, 1997) recurrent neural network language model (RNNLM; Mikolov et al., 2010). Unlike the model in this paper, which only considers the story endings, this language model follows the protocol suggested by the story cloze task designers, and harnesses their ROC Stories training set, which consists of single-ending stories, as well as the story context for each pair of endings. We show that adding our features to this powerful language model gives improvements over our classifier as well as the language model.

We train the RNNLM using a single-layer LSTM of hidden dimension 512. We use the ROC stories for training, ${ }^{9}$ setting aside $10 \%$ for validation of the language model. We replace all words occurring less than 3 times with an outof-vocabulary token, yielding a vocabulary size of 21,582 . Only during training, we apply a dropout rate of $60 \%$ while running the LSTM over all 5 sentences of the stories. Using the Adam optimizer (Kingma and $\mathrm{Ba}, 2015$ ) and a learning rate of $\eta=0.001$, we train to minimize cross-entropy.

To apply the language model to the classification problem, we select as right the ending with the higher value of

$$
\frac{p_{\theta}(\text { ending } \mid \text { story })}{p_{\theta}(\text { ending })}
$$

\footnotetext{
${ }^{9}$ We use the extended, 100K stories corpus (see Section 2).
} 


\begin{tabular}{|l|c|}
\hline Feature Type & Accuracy \\
\hline Word $n$-grams & 0.612 \\
\hline Character $n$-grams & 0.639 \\
\hline Full model & 0.645 \\
\hline
\end{tabular}

Table 4: Results on Experiment 1 with different subsets of features.

The intuition is that a right ending should be unsurprising (to the model) given the four preceding sentences of the story (the numerator), controlling for the inherent surprisingness of the words in that ending (the denominator).

On its own, our neural language model performs moderately well on the story cloze test. Selecting endings based on $p_{\theta}$ (ending | story) (i.e., the numerator of Equation 1), we obtained only $55 \%$ accuracy. The ratio in Equation 1 achieves $67.7 \%$ (see Table 3). ${ }^{10}$

We combine our linear model with the RNNLM by adding three features to our classifier: the numerator, denominator, and ratio in Equation 1, all in log space. We retrain our linear model with the new feature set, and gain $2.8 \%$ absolute, reaching $75.2 \%$, a new state of the art result for the task. These results indicate that context-ignorant style features can be used to obtain high accuracy on the task, adding value even when context and a large training dataset are used.

\section{Further Analysis}

\subsection{Most Discriminative Feature Types}

A natural question that follows from this study is which style features are most helpful in detecting the underlying task an author was asked to perform. To answer this question, we re-ran Experiment 1 with different sub-groups of features. Table 4 shows our results. Results show that character $n$-grams are the most effective style predictors, reaching within $0.6 \%$ of the full model, but that word $n$-grams also capture much of the signal, yielding $61.2 \%$, which is only $3.3 \%$ worse than the full model. These findings are in line with previous work that used character $n$-grams along with other types of features to predict writing style (Schwartz et al., 2013b).

\footnotetext{
${ }^{10}$ Note that taking the logarithm of the expression in Equation 1 gives the pointwise mutual information between the story and the ending, under the language model.
}

\subsection{Most Salient Features}

A follow-up question is which individual features contribute most to the classification process, as these could shed light on the stylistic differences imposed by each of the writing tasks.

In order to answer this question, we consider the highest absolute positive and negative coefficients in the logistic regression classifier in Experiments 1 and 2, an approach widely used as a method of extracting the most salient features (Nguyen et al., 2013; Burke et al., 2013; Brooks et al., 2013). It is worth noting that its reliability is not entirely clear, since linear models like logistic regression can assign large coefficients to rare features (Yano et al., 2012). To mitigate this concern, we consider only features appearing in at least $5 \%$ of the endings in our training set.

Experiment 1. Table 5a shows the most salient features for right (coherent) and wrong (incoherent) endings in Experiment 1, along with their corpus frequency. The table shows a few interesting trends. First, authors tend to structure their sentences differently when writing coherent vs. incoherent endings. For instance, incoherent endings are more likely to start with a proper noun and end with a common noun, while coherent endings have a greater tendency to end with a past tense verb.

Second, right endings make wider use of coordination structures, as well as adjectives. The latter might indicate that writing coherent stories inspires the authors to write more descriptive text compared to incoherent ones, as is the case in truthful vs. deceptive text (Ott et al., 2011). Finally, we notice a few syntactic differences: right endings more often use infinitive verb structure, while wrong endings prefer gerunds (VBG).

Experiment 2. Table 5b shows the same analysis for Experiment 2. As noted in Section 2, original endings tend to be much longer, which is indeed the most salient feature for them. An interesting observation is that exclamation marks are a strong indication for an original ending. This suggests that authors are more likely to show or evoke enthusiasm when writing their own text compared to ending an existing text.

Finally, when comparing the two groups of salient features from both experiments, we find an interesting trend. Several features, such as "START NNP" and "NN .", which indicate wrong sentences in Experiment 1, are used to predict 


\begin{tabular}{|c|c|c||c|c|c|}
\hline Right & Weight & Freq. & Wrong & Weight & Freq. \\
\hline 'ed .' & 0.17 & $6.5 \%$ & START NNP & 0.21 & $54.8 \%$ \\
\hline 'and ' & 0.15 & $13.6 \%$ & NN . & 0.17 & $47.5 \%$ \\
\hline JJ & 0.14 & $45.8 \%$ & NN NN . & 0.15 & $5.1 \%$ \\
\hline to VB & 0.13 & $20.1 \%$ & VBG & 0.11 & $10.1 \%$ \\
\hline 'd th' & 0.12 & $10.9 \%$ & START NNP VBD & 0.11 & $41.9 \%$ \\
\hline
\end{tabular}

(a) Experiment 1

\begin{tabular}{|c|c|c||c|c|c|}
\hline Right & Weight & Freq. & Wrong & Weight & Freq. \\
\hline length & 0.81 & $100.0 \%$ & $\ddots$ & 0.74 & $93.0 \%$ \\
\hline '? & 0.46 & $6.1 \%$ & START NNP & 0.40 & $39.2 \%$ \\
\hline NN & 0.35 & $78.9 \%$ & START NNP VBD & 0.23 & $29.0 \%$ \\
\hline RB & 0.34 & $44.7 \%$ & NN . & 0.20 & $42.3 \%$ \\
\hline,, & 0.32 & $12.7 \%$ & the NN . & 0.20 & $10.6 \%$ \\
\hline
\end{tabular}

(b) Experiment 2

Table 5: The top 5 most heavily weighted features for predicting right vs. wrong endings (5a) and original vs. new (right) endings (5b). length is the sentence length feature (see Section 4).

new (i.e., right) endings in Experiment 2. This indicates that, for instance, incoherent endings have a stronger tendency to begin with a proper noun compared to coherent endings, which in turn are more likely to do so than original endings. This partially explains why distinguishing between original and wrong endings is an easier task compared to the other pairs (Section 5.1).

\section{Discussion}

The effect of writing tasks on mental states. In this paper we have shown that different writing tasks affect a writer's writing style in easily detected ways. Our results indicate that when authors are asked to write the last sentence of a fivesentence story, they will use different style to write a right ending compared to a wrong ending. We have also shown that writing the ending as part of one's own five-sentence story is very different than reading four sentences and then writing the fifth. Our findings hint that the nature of the writing task imposes a different mental state on the author, which is expressed in ways that can be observed using extremely simple automatic tools.

Previous work has shown that a writing task can affect mental state. For instance, writing deceptive text leads to a significant cognitive burden accompanied by a writing style that is different from truthful text (Newman et al., 2003; Banerjee et al., 2014). Writing tasks can even have a long-term effect, as writing emotional texts was observed to benefit both physical and mental health (Lep- ore and Smyth, 2002; Frattaroli, 2006). Campbell and Pennebaker (2003) also showed that the health benefits of writing emotional text are accompanied by changes in writing style, mostly in the use of pronouns.

Another line of work has shown that writing style is affected by mental state. First, an author's personality traits (e.g., depression, neuroticism, narcissism) affect her writing style (Schwartz et al., 2013a; Ireland and Mehl, 2014). Second, temporary changes, such as a romantic relationship (Ireland et al., 2011; Bowen et al., 2016), work collaboration (Tausczik, 2009; Gonzales et al., 2009), or negotiation (Ireland and Henderson, 2014) may also affect writing style. Finally, writing style can also change from one sentence to another, for instance between positive and negative text (Davidov et al., 2010) or when writing sarcastic text (Tsur et al., 2010).

This large body of work indicates a tight connection between writing tasks, mental states, and variation in writing style. This connection hints that the link discovered in this paper, between different writing tasks and resulting variation in writing style, involves differences in mental state. Additional investigation is required in order to further validate this hypothesis.

Design of NLP tasks. Our study also provides important insights for the future design of NLP tasks. The story cloze task was very carefully designed. Many factors, such as topic diversity and 
temporal and causal relation diversity, were controlled for (Mostafazadeh et al., 2016a). The authors also made sure each pair of endings was written by the same author, partly in order to avoid author-specific style effects. Nonetheless, despite these efforts, several significant style differences can be found between the story cloze training and test set, as well as between the positive and negative labels.

Our findings suggest that careful attention must be paid to instructions given to authors, especially in unnatural tasks such as writing a wrong ending. The COPA dataset (Roemmele et al., 2011), which was also designed to test commonsense knowledge, explicitly addressed potential style differences in their instructions. In this task, systems are presented with premises like I put my plate in the sink, and then decide between two alternatives, e.g.: (a) I finished eating. and (b) I skipped dinner. Importantly, when writing the alternatives, annotators were asked to be as brief as possible and avoid proper names, as well as slang.

Applying our story cloze classifier to this dataset yields $53.2 \%$ classification accuracyclose to a random baseline. While this could be partially explained by the smaller data size of the COPA dataset (1,000 examples compared to 3,742 in the story cloze task), this indicates that simple instructions may help alleviate the effects of writing style found in this paper. Another way to avoid such effects is to have people rate naturally occurring sentences by parameters such as coherence (or, conversely, the level of surprise), rather than asking them to generate new text.

\section{Related Work}

Writing style. Writing style has been an active topic of research for decades. The models used to characterize style are often linear classifiers with style features such as character and word $n$-grams (Stamatatos, 2009; Koppel et al., 2009). Previous work has shown that different authors can be grouped by their writing style, according to factors such as age (Pennebaker and Stone, 2003; Argamon et al., 2003; Schler et al., 2006; Rosenthal and McKeown, 2011; Nguyen et al., 2011), gender (Argamon et al., 2003; Schler et al., 2006; Bamman et al., 2014), and native language (Koppel et al., 2005; Tsur and Rappoport, 2007; Bergsma et al., 2012). At the extreme case, each individual author adopts a unique writing style (Mosteller and Wallace, 1963; Pennebaker and King, 1999; Schwartz et al., 2013b).

The line of work that most resembles our work is the detection of deceptive text. Several researchers have used stylometric features to predict deception (Newman et al., 2003; Hancock et al., 2007; Ott et al., 2011; Feng et al., 2012). Some works even showed that gender affects a person's writing style when lying (Pérez-Rosas and Mihalcea, 2014a,b). In this work, we have shown that an even more subtle writing task-writing coherent and incoherent story endings-imposes different styles on the author.

Machine reading. The story cloze task, which is the focus of this paper, is part of a wide set of machine reading/comprehension challenges published in the last few years. These include datasets like bAbI (Weston et al., 2016), SNLI (Bowman et al., 2015), CNN/DailyMail (Hermann et al., 2015), LAMBADA (Paperno et al., 2016) and SQuAD (Rajpurkar et al., 2016). While these works have presented resources for researchers, it is often the case that these datasets suffer from methodological problems caused by applying noisy automatic tools to generate them (Chen et al., 2016). ${ }^{11}$ In this paper, we have pointed to another methodological challenge in designing machine reading tasks: different writing tasks used to generated the data affect writing style, confounding classification problems.

\section{Conclusion}

Different writing tasks assigned to an author result in different writing styles for that author. We experimented with the story cloze task, which introduces two interesting comparison points: the difference between writing a story on one's own and continuing someone else's story, and the difference between writing a coherent and an incoherent story ending. In both cases, a simple linear model reveals measurable differences in writing styles, which in turn allows our final model to achieve state of the art results on the story cloze task.

The findings presented in this paper have cognitive implications, as they motivate further research

\footnotetext{
${ }^{11}$ Similar problems have been shown in visual question answering datasets, where simple models that rely mostly on the question text perform competitively with state of the art models by exploiting language biases (Zhou et al., 2015; Jabri et al., 2016).
} 
on the effects that a writing prompt has on an author's mental state, and also her concrete response. They also provide valuable lessons for designing new NLP datasets.

\section{Acknowledgments}

The authors thank Chenhao Tan, Luke Zettlemoyer, Rik Koncel-Kedziorski, Rowan Zellers, Yangfeng Ji and several anonymous reviewers for helpful feedback. This research was supported in part by Darpa $\mathrm{CwC}$ program through $\mathrm{ARO}$ (W911NF-15-1-0543), Samsung GRO, NSF IIS1524371, and gifts from Google and Facebook.

\section{References}

Shlomo Argamon, Moshe Koppel, Jonathan Fine, and Anat Rachel Shimoni. 2003. Gender, genre, and writing style in formal written texts. Text 23(3):321346.

David Bamman, Jacob Eisenstein, and Tyler Schnoebelen. 2014. Gender identity and lexical variation in social media. Journal of Sociolinguistics 18(2):135160.

Ritwik Banerjee, Song Feng, Jun S. Kang, and Yejin Choi. 2014. Keystroke patterns as prosody in digital writings: A case study with deceptive reviews and essays. In Proc. of EMNLP.

Shane Bergsma, Matt Post, and David Yarowsky. 2012. Stylometric analysis of scientific articles. In Proc. of NAACL.

Jeffrey D. Bowen, Lauren A. Winczewski, and Nancy L. Collins. 2016. Language style matching in romantic partners? conflict and support interactions. Journal of Language and Social Psychology pages $1-24$.

Samuel R. Bowman, Gabor Angeli, Christopher Potts, and Christopher D. Manning. 2015. A large annotated corpus for learning natural language inference. In Proc. of EMNLP.

Michael Brooks, Katie Kuksenok, Megan K. Torkildson, Daniel Perry, John J. Robinson, Taylor J. Scott, Ona Anicello, Ariana Zukowski, Paul Harris, and Cecilia R. Aragon. 2013. Statistical affect detection in collaborative chat. In Proc. of CSCW.

Moira Burke, Lada A. Adamic, and Karyn Marciniak. 2013. Families on facebook. In Proc. of ICWSM.

Zheng Cai, Lifu Tu, and Kevin Gimpel. 2017. Pay attention to the ending: Strong neural baselines for the ROC story cloze task. In Proc. of ACL.

R. Sherlock Campbell and James W. Pennebaker. 2003. The secret life of pronouns flexibility in writing style and physical health. Psychological Science 14(1):60-65.

Danqi Chen, Jason Bolton, and Christopher D. Manning. 2016. A thorough examination of the CNN/Daily Mail reading comprehension task. In Proc. of ACL.

Dmitry Davidov, Oren Tsur, and Ari Rappoport. 2010. Enhanced sentiment learning using Twitter hashtags and smileys. In Proc. of COLING.

Song Feng, Ritwik Banerjee, and Yejin Choi. 2012. Syntactic stylometry for deception detection. In Proc. of ACL.

Joanne Frattaroli. 2006. Experimental disclosure and its moderators: a meta-analysis. Psychological bulletin 132(6):823.

Amy L. Gonzales, Jeffrey T. Hancock, and James W. Pennebaker. 2009. Language style matching as a predictor of social dynamics in small groups. Communication Research 37(1):3-19.

Jeffrey T. Hancock, Lauren E. Curry, Saurabh Goorha, and Michael Woodworth. 2007. On lying and being lied to: A linguistic analysis of deception in computer-mediated communication. Discourse Processes 45(1):1-23.

Karl Moritz Hermann, Tomáš Kočiský, Edward Grefenstette, Lasse Espeholt, Will Kay, Mustafa Suleyman, and Phil Blunsom. 2015. Teaching machines to read and comprehend. In Proc. of NIPS.

Sepp Hochreiter and Jürgen Schmidhuber. 1997. Long short-term memory. Neural Computation 9(8):1735-1780.

Molly E. Ireland and Marlone D. Henderson. 2014. Language style matching, engagement, and impasse in negotiations. Negotiation and Conflict Management Research 7(1):1-16.

Molly E. Ireland and Matthias R. Mehl. 2014. Natural language use as a marker of personality, Oxford University Press, USA, pages 201-237. The Oxford Handbook of Language and Social Psychology.

Molly E. Ireland, Richard B. Slatcher, Paul W. Eastwick, Lauren E. Scissors, Eli J. Finkel, and James W. Pennebaker. 2011. Language style matching predicts relationship initiation and stability. Psychological Science 22(1):39-44.

Allan Jabri, Armand Joulin, and Laurens van der Maaten. 2016. Revisiting visual question answering baselines. In Proc. of ECCV.

Diederik Kingma and Jimmy Ba. 2015. Adam: A method for stochastic optimization. In Proc. of ICLR.

Moshe Koppel, Jonathan Schler, and Shlomo Argamon. 2009. Computational methods in authorship attribution. Journal of the American Society for information Science and Technology 60(1):9-26. 
Moshe Koppel, Jonathan Schler, and Kfir Zigdon. 2005. Determining an author's native language by mining a text for errors. In Proc. of KDD.

Stephen J. Lepore and Joshua M. Smyth. 2002. The Writing Cure: How Expressive Writing Promotes Health and Emotional Well-being. American Psychological Association.

Todor Mihaylov and Anette Frank. 2017. Simple story ending selection baselines. In Proc. of LSDSem.

Tomáš Mikolov, Martin Karafiát, Lukáš Burget, Jan Černocký, and Sanjeev Khudanpur. 2010. Recurrent neural network based language model. In Proc. of Interspeech.

Nasrin Mostafazadeh, Nathanael Chambers, Xiaodong He, Devi Parikh, Dhruv Batra, Lucy Vanderwende, Pushmeet Kohli, and James Allen. 2016a. A corpus and cloze evaluation for deeper understanding of commonsense stories. In Proc. of NAACL.

Nasrin Mostafazadeh, Michael Roth, Annie Louis, Nathanael Chambers, and James F. Allen. 2017. LSDSem 2017 shared task: The story cloze test. In Proc. of LSDSem.

Nasrin Mostafazadeh, Lucy Vanderwende, Wen-tau Yih, Pushmeet Kohli, and James Allen. 2016b. Story cloze evaluator: Vector space representation evaluation by predicting what happens next. In Proc. of RepEval.

Frederick Mosteller and David L. Wallace. 1963. Inference in an authorship problem. Journal of the American Statistical Association 58(302):275-309.

Matthew L. Newman, James W. Pennebaker, Diane S. Berry, and Jane M. Richards. 2003. Lying words: Predicting deception from linguistic styles. Personality and Social Psychology Bulletin 29(5):665-675.

Dong Nguyen, Rilana Gravel, Dolf Trieschnigg, and Theo Meder. 2013. "How old do you think i am?" a study of language and age in Twitter. In Proc. of ICWSM.

Dong Nguyen, Noah A. Smith, and Carolyn P. Rosé. 2011. Author age prediction from text using linear regression. In Proc. of $\mathrm{LaTeCH}$.

Myle Ott, Yejin Choi, Claire Cardie, and Jeffrey T. Hancock. 2011. Finding deceptive opinion spam by any stretch of the imagination. In Proc. of $A C L$.

Denis Paperno, Germán Kruszewski, Angeliki Lazaridou, Ngoc Quan Pham, Raffaella Bernardi, Sandro Pezzelle, Marco Baroni, Gemma Boleda, and Raquel Fernández. 2016. The LAMBADA dataset: Word prediction requiring a broad discourse context. In Proc. of ACL.

Fabian Pedregosa, Gaël Varoquaux, Alexandre Gramfort, Vincent Michel, Bertrand Thirion, Olivier Grisel, Mathieu Blondel, Peter Prettenhofer, Ron
Weiss, Vincent Dubourg, Jake Vanderplas, Alexandre Passos, David Cournapeau, Matthieu Brucher, Matthieu Perrot, and Édouard Duchesnay. 2011. Scikit-learn: Machine learning in Python. JMLR $12: 2825-2830$.

James W. Pennebaker and Laura A. King. 1999. Linguistic styles: language use as an individual difference. Journal of Personality and Social Psychology 77(6):1296-1312.

James W. Pennebaker and Lori D. Stone. 2003. Words of wisdom: language use over the life span. Journal of Personality and Social Psychology 85(2):291301.

Verónica Pérez-Rosas and Rada Mihalcea. 2014a. Cross-cultural deception detection. In Proc. of ACL.

Verónica Pérez-Rosas and Rada Mihalcea. 2014b. Gender differences in deceivers writing style. Lecture Notes in Computer Science 8856:163-174.

Tiantian Qin, Judee Burgoon, and Jay F. Nunamaker Jr. 2004. An exploratory study on promising cues in deception detection and application of decision tree. In Proc. of HICSS.

Pranav Rajpurkar, Jian Zhang, Konstantin Lopyrev, and Percy Liang. 2016. SQuAD: 100,000+ questions for machine comprehension of text. In Proc. of EMNLP.

Melissa Roemmele, Cosmin Adrian Bejan, and Andrew S. Gordon. 2011. Choice of plausible alternatives: An evaluation of commonsense causal reasoning. In AAAI Spring Symposium: Logical Formalizations of Commonsense Reasoning.

Sara Rosenthal and Kathleen McKeown. 2011. Age prediction in blogs: A study of style, content, and online behavior in pre- and post-social media generations. In Proc. of ACL.

Michael Roth, Nasrin Mostafazadeh, Nathanael Chambers, and Annie Louis, editors. 2017. Proceedings of the 2nd Workshop on Linking Models of Lexical, Sentential and Discourse-level Semantics. Association for Computational Linguistics.

Jonathan Schler, Moshe Koppel, Shlomo Argamon, and James Pennebaker. 2006. Effects of age and gender on blogging. In AAAI Spring Symposium: Computational Approaches to Analyzing Weblogs.

Andrew H. Schwartz, Johannes C. Eichstaedt, Margaret L. Kern, Lukasz Dziurzynski, Stephanie M. Ramones, Megha Agrawal, Achal Shah, Michal Kosinski, David Stillwell, Martin E.P. Seligman, and Lyle H. Unger. 2013a. Personality, gender, and age in the language of social media: The openvocabulary approach. PloS one 8(9):e73791.

Roy Schwartz, Maarten Sap, Ioannis Konstas, Leila Zilles, Yejin Choi, and Noah A. Smith. 2017. Story cloze task: UW NLP system. In Proc. of LSDSem. 
Roy Schwartz, Oren Tsur, Ari Rappoport, and Moshe Koppel. 2013b. Authorship attribution of micromessages. In Proc. of EMNLP.

Efstathios Stamatatos. 2009. A survey of modern authorship attribution methods. Journal of the American Society for information Science and Technology 60(3):538-556.

Yla Rebecca Tausczik. 2009. Linguistic analysis of workplace computer-mediated communication. Master's thesis, University of Texas.

Oren Tsur, Dmitry Davidov, and Ari Rappoport. 2010. ICWSM - a great catchy name: Semi-supervised recognition of sarcastic sentences in online product reviews. In Proc. of ICWSM.

Oren Tsur and Ari Rappoport. 2007. Using classifier features for studying the effect of native language on the choice of written second language words. In Proc. of CACLA.

Jason Weston, Antoine Bordes, Sumit Chopra, Alexander M Rush, Bart van Merriënboer, Armand Joulin, and Tomas Mikolov. 2016. Towards AI-complete question answering: A set of prerequisite toy tasks. In Proc. of ICLR.

Maria Yancheva and Frank Rudzicz. 2013. Automatic detection of deception in child-produced speech using syntactic complexity features. In Proc. of ACL.

Tae Yano, Noah A. Smith, and John D. Wilkerson. 2012. Textual predictors of bill survival in congressional committees. In Proc. of NAACL.

Bolei Zhou, Yuandong Tian, Sainbayar Sukhbaatar, Arthur Szlam, and Rob Fergus. 2015. Simple baseline for visual question answering. ArXiv:1512.02167. 